Intensivmed 2010 • 47:81-82

DOI 10.1007/s00390-009-0141-1

Online publiziert: 6. März 2010

(c) Springer-Verlag 2010

C. Dodt

Präklinik, Städtisches Klinikum München-Bogenhausen

\title{
Leitsymptom Bewusstseinsstörung
}

hen deutlich über das hinaus, was derzeit in Deutschland an Ausbildungsinhalten für die Zusatzbezeichnung „Notfallmedizin“ gefordert ist, die primär als Qualifikationsnachweis für präklinisch tätige Notfallmediziner dient.

Die Zeitschrift „Intensivmedizin und Notfallmedizin" widmet sich traditionell der Weiterbildung in der Notfallmedizin und greift immer wieder Themen aus den Bereichen auf, die die Intensivmedizin und die Notfallmedizin in gleichem Maße betreffen. Das aktuelle Themenheft beschäftigt sich mit den „Bewusstseinsstörungen". Diese sind in Rettungsdienst und Notaufnahmen sehr häufig anzutreffen und müssen von den Behandelnden als ein Warnsymptom, das besonders dringlicher Abklärung bedarf, wahrgenommen werden. Wenn die Funktionen der verschiedenen Organsysteme so eingeschränkt sind, dass eine regelrechte Funktion des Endorgans Gehirn nicht mehr gewährleistet ist, ist das ebenso lebensbedrohlich wie der primäre Ausfall zentralnervöser Funktionen, die die Wachheit garantieren. In diesen Fällen ist ein sehr strukturiertes und rasches Vorgehen notwendig, um prinzipiell behebbare Ursachen von Bewusstseinsstörungen rasch zu beseitigen. Jedes Krankenhaus kann dabei die Qualität seiner Notfalltherapie anhand der Tracerdiagnose „Verschluss der A. basilaris“ selbst kontrollieren. Sind die diagnostischen Prozesse bei den Patienten mit dem Symptom "Bewusstseinsstörung“ optimal, findet eine therapeutische Intervention innerhalb von $3 \mathrm{~h}$ nach Auftreten der Symptome statt. Ist dies nicht der Fall, muss sich jedes Krankenhaus ernsthafte Gedanken um die Prozessqualität im Bereich der Akutaufnahme machen.

Innerhalb der Notaufnahmesituation gilt es, die lebensbedrohlichen und therapier- baren Störungen des Bewusstseins rasch zu erfassen.

Dabei muss fachübergreifend an primäre Störungen des Zentralnervensystems ebenso gedacht werden wie an internistisch-metabolische Störungen und Intoxikationen. All diese Aspekte werden in einer zentralen Notaufnahme zusammengefasst, und natürlich darf dabei auch nicht vergessen werden, dass ein plötzlicher Bewusstseinsverlust oft Sturzfolgen nach sich zieht, die ebenfalls in der Aufnahmesituation besonderer Aufmerksamkeit bedürfen.

Ich hoffe, dass in diesem Themenheft ein umfassender Themenüberblick gelungen ist, der hilft, bei unseren Patienten rasch das Bewusstsein wieder herzustellen. Gleichzeitig soll das Bewusstsein der Leser dafür erweitert werden, dass Notfallmedizin so attraktiv ist, weil sie fachübergreifendes Arbeiten erfordert.

Vergessen Sie nicht: „Der Schlaf ist des Todes Bruder“!
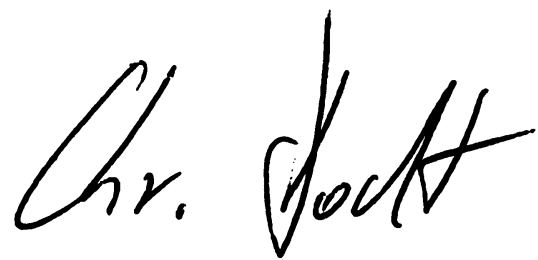

\section{Dodt}

\section{Korrespondenzadresse Prof. Dr. C. Dodt}

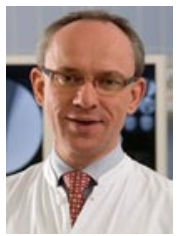

Präklinik, Städtisches Klinikum München-Bogenhausen Englschalkinger Straße 77, 81925 München christoph.dodt@ kh-bogenhausen.de dungscurriculum der European Society of Emergency Medicine (EuSEM) orientieren. Diese Ausbildungsempfehlungen ge- 


\section{Michels/Kochanek \\ Repetitorium internistische Intensivmedizin}

Springer-Verlag GmbH, 2010, ca. 508 S., 72

Abb., LP 39,95€, ISBN: 978-3-642-02719-2

\section{Wissen, worauf es ankommt!}

Die Kombination der beiden großen Fächer „Innere Medizin“ und „Intensivmedizin“ ist eine Herausforderung an Wissen und Kön-

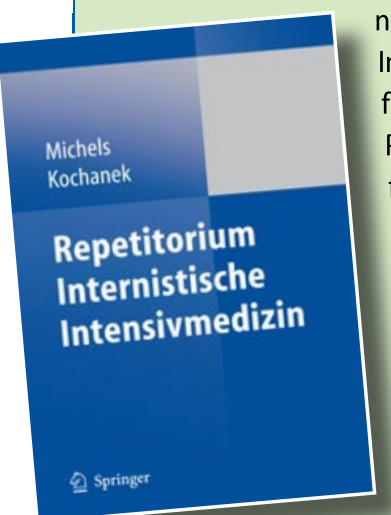
nen! Doch welche Informationen sind für Verständnis, Praxis und Prüfung tatsächlich wichtig? Im Repetitorium können angehende und auch bereits tätige Intensivmediziner nachlesen, wor-

auf es wirklich ankommt. Erfahrene Intensivmediziner stellen das gesamte Spektrum des Fachgebietes praxisnah, übersichtlich und kompakt dar. Aufgrund der vielen Praxis-Tipps sowie Übersichten und Tabellen ist das Buch sowohl für die Klinikalltag als auch die Prüfung bestens geeignet. Für alle Ärzte auf internistischen Intensivstationen zum schnellen Nachschlagen im Klinikalltag oder zum Lernen für die Prüfung.

\section{Interview mit den Herausgebern Herrn Dr. Michels und Herrn Dr. Kochanek}

\section{? Was ist das Besondere an Ihrem} Buch?

Dr. Michels: Die internistische Intensivmedizin ist ein Komplex aus Innerer Medizin, Notfallmedizin, Anästhesie und Neurologie. Das Besondere an unserem Buch ist, dass alle diese Themenkomplexe besprochen werden - von der Erstellung eines Ernährungsplans, der Durchführung einer Reanimation, der Erstellung eines "Weaning-Protokolls" bis zur Veranlassung einer Hirntoddiagnostik. Wir haben besonderen Wert darauf gelegt, dass es viele konkrete Angaben zum genauen Vorgehen gibt. Darüber hinaus werden die Inhalte mit zahlreichen Tipps und Tricks von prak- tizierenden Intensivmedizinern "gewürzt", so dass Theorie und Praxis in idealer Weise verknüpft werden. Uns war auch wichtig, dass die Inhalte nachvollziehbar sind und verstanden werden. Das erleichtert die Arbeit enorm. Stures Auswendiglernen macht keinen guten Intensivmediziner.

\section{? An wen richtet sich das Buch?}

Dr. Michels: Das Repetitorium Internistische Intensivmedizin richtet sich an alle intensivmedizinisch tätigen Kolleginnen und Kollegen, insbesondere an diejenigen, die im Rahmen der Ausbildung zum Facharzt für "Innere Medizin“ oder "Innere Intensivmedizin“ auf einer Intensivstation arbeiten. Ein Teil unserer Autoren gehört zu dieser Zielgruppe. So konnten wir auf die Bedürfnisse direkt eingehen und Probleme bzw. Fragestellungen aus der täglichen Arbeit direkt mit aufnehmen.

? Sind die Inhalte des Repetitoriums auf alle Intensivstationen in Deutschland übertragbar?

Dr. Kochanek: Das hängt vom Versorgungsstatus der Klinik ab - Haus der Minimal- versus Maximalversorgung. Natürlich spielen die Voraussetzungen und Ressourcen einer Klinik, d.h. Fachpflegepersonal, Dialysemöglichkeiten, Herzkatheterlabor etc. eine Rolle. Aber im Prinzip sind die Inhalte auf alle Intensivstationen übertragbar. Viele der Diagnostik- und Therapiemethoden sind von logistischen oder personellen Voraussetzungen unabhängig.

? Haben Sie eine Strategie für die optimale Behandlung Ihrer Patienten?

Dr. Kochanek: Es gibt verschiedene Strategien. Neben der klinischen Erfahrung, die extrem wichtig ist, kann durch regelmäßige klinikinterne oder -externe Weiterbildung oder "autodidaktische" Fortbildung, z.B. durch Nachlesen ein sicherer Umgang mit Intensivpatienten erreicht werden. Eine Garantie dafür, dass alles klappt, gibt es nicht. $100 \%$ ig vorbereiten kann man sich nicht, da jeder Patient, jede Situation ein wenig anders ist. Mit der Erfahrung wächst glücklicherweise das Gespür, auch für kritische Situationen.

? Nach welcher Systematik können Kollegen für eine erfolgreiche Prüfung lernen?

Dr. Michels: Das Repetitorium bietet eine optimale Prüfungsvorbereitung. In den beiden Hauptkategorien - allgemeine und spezielle Intensivmedizin - werden alle wichtigen und prüfungsrelevanten Themen didaktisch, leitliniengerecht und hochaktuell auf den Punkt gebracht. Der Repetitorium-Stil garantiert dabei den Blick aufs Wesentliche und absolute Praxisnähe. Aber auch das Verständnis kommt nicht zu kurz. Die Physiologie spielt dabei eine wichtige Rolle. Ohne deren Verständnis ist gute Intensivmedizin nicht möglich.

Welchen Tipp können Sie Kollegen geben, die die Intensivstation noch vor sich haben?

Dr. Kochanek: Um nach kurzer Zeit eine gewisse „Sicherheit" auf Intensivstation zu erlangen, sind im Grunde drei Dinge wichtig: erstens - ein guter Mentor, der mit Rat und Tat zur Seite steht, zweitens - ein gutes Buch zum Nachschlagen und Lernen und drittens - Intensivschwestern und Intensivpfleger, die ihr Wissen und ihren Erfahrungsschatz mit Ihnen teilen.

Vielen Dank für das Gespräch!

Dr. Anna Krätz,

Springer Medizin Heidelberg 